\title{
Dynamical Localization of the Hofstadter Spectra
}

\author{
P. H. Rivera* and P. A. Schulz ${ }^{\dagger}$ \\ ${ }^{*}$ Consejo Superior de Investigaciones, Facultad de Ciencias Físicas, \\ Universidad Nacional Mayor de San Marcos, Lima, Perú and \\ ${ }^{\dagger}$ Instituto de Física “Gleb Wataghin”, Universidade Estadual de Campinas, 13083-970, Campinas, São Paulo, Brasil
}

Received on 4 April, 2005

\begin{abstract}
Recent results on magnetoresistance in a two dimensional electron gas (2DEG) under crossed magnetic and microwave fields show a new class of oscillations, suggesting a new kind of zero-resistance states. We consider the problem from the point of view of the electronic structure dressed by photons due to an in-plane linearly polarized ac field. In the strong modulation limit predictions on dressed Hofstadter spectra are discussed, which could be of interest since the bare spectra have been observed in the past few years.
\end{abstract}

Keywords: Dynamical localization; Hofstadter spectra; (2DEG)

A two dimensional electron gas (2DEG) under an intense and perpendicular magnetic field shows oscillations with some zero resistance in the longitudinal magnetoresistance that coincide with plateaus in the transverse magnetoresistance for some ranges of magnetic field values. This was named the integer quantum Hall effect[1]. A few years ago, in high mobility 2 DEG at very low magnetic field and with an applied microwave radiation along the plane showed zero resistance $(\mathrm{ZR})$ values in the longitudinal magnetoresistance, which are not observed in the absence of the ac field radiation[2, 3].

These experimental observations have generated an avalanche of theoretical works to explain these ZR states induced by radiation. An interesting topic to investigate is to include a periodic potential modulation to the 2DEG[4] and the purpose of this work is to obtain some insights to this problem. We know that these experimental observations occur at $B<0.2 \mathrm{~T}$ and radiation frequency $\mathrm{v}<100 \mathrm{GHz}$. Due to dimensions of the samples and the intensity of the radiation, as was explained in a previous paper[5], these fenomena cannot be explained perturbatively. We address the problem from dressed electronic structures point of view, developing a tight binding model coupled nonperturbatively to ac field including a magnetic field.

The spectra of a bare electronic system are described by a heuristic tight binding square lattice of $s$-orbitals with nearestneighbor interaction. The magnetic field interacting with the system is introduced by means of the Peierls substitution. Choosing the Landau gauge $\mathbf{A}=\left(0, l_{1} a B, 0\right)$ leads to the following Hamiltonian

$$
\begin{gathered}
H_{0}=\sum_{l_{1}, l_{2}} \varepsilon_{l_{1}, l_{2}} \sigma_{l_{1}, l_{2}} \sigma_{l_{1}, l_{2}}^{\dagger}+\frac{V}{2} \sum_{l_{1}, l_{2}}\left[\sigma_{l_{1}, l_{2}} \sigma_{l_{1}+1, l_{2}}^{\dagger}+\right. \\
\left.\sigma_{l_{1}+1, l_{2}} \sigma_{l_{1}, l_{2}}^{\dagger}+e^{i 2 \pi \alpha l_{1}}\left(\sigma_{l_{1}, l_{2}} \sigma_{l_{1}, l_{2}+1}^{\dagger}+\sigma_{l_{1}, l_{2}+1} \sigma_{l_{1}, l_{2}}^{\dagger}\right)\right],
\end{gathered}
$$

here $\sigma_{l_{1}, l_{2}}=\left|l_{1}, l_{2}\right\rangle$ and $\sigma_{l_{1}, l_{2}}^{\dagger}=\left\langle l_{1}, l_{2}\right|$ where $\left(l_{1}, l_{2}\right)$ are the $(x, y)$ coordinates of the sites. The atomic energies are taken constant, $\varepsilon_{l_{1}, l_{2}}=4|V|$ for all sites. For an antidot lattice[6], we consider $\varepsilon_{l_{1}, l_{2}}=4|V|$ in host lattice and $\varepsilon_{l_{1}, l_{2}}=8|V|$ in antidot sites, while $V$, the nearest-neighbor hopping parameter, is kept constant to $V=-\hbar^{2} / 2 m^{*} a^{2}$ in both $x$ and $y$ directions. The lattice parameter of the antidot array is $a^{\prime}=n a$, where $a$ is the host lattice constant, and the lateral dimension of the array is $L=m a^{\prime}$. The surface of the antidot array is defined by hard wall boundary conditions.

The magnetic phase factor $\alpha$ is defined by $\alpha=\Phi / \Phi_{e}=$ $p / q$, where $\Phi_{e}=h / e$ is the magnetic flux quantum, and $\Phi=$ $a^{2} B$ is the magnetic flux per unit cell of the host lattice. The magnetic flux through an antidot unit cell is given by $\Phi^{\prime}=$ $n^{2} \Phi$, and the magnetic flux through the total array is $\Phi^{T}=$ $n^{2} m^{2} \Phi[6]$.

An ac field is applied parallel to one of the square lattice sides. The coupling of the system to an arbitrarily intense ac field is described by the Hamiltonian $H=H_{0}+H_{\text {int }}$, where

$$
H_{i n t}=e a F \cos \omega t \sum_{l_{1}, l_{2}} \sigma_{l_{1}, l_{2}} l_{1} \sigma_{l_{1}, l_{2}}^{\dagger}
$$

the ac field is parallel to $x$ axis as can be seen by the linear dependence in $l_{1}, e$ is the electron charge, $\omega$ and $F$ are the monochromatic ac field frequency and amplitude, respectively. The treatment of the time-dependent problem is based on Floquet states $\left|l_{1}, l_{2}, k\right\rangle$ where $k$ is the photon index. We follow the procedure developed by Shirley[7], which consist in a Fourier-Floquet transformation of the time-dependent Hamiltonian into a time-independent infinity matrix. The elements of this infinite matrix are

$$
\begin{gathered}
{\left[\left(\mathcal{E}-k \hbar \omega-\varepsilon_{l_{1}, l_{2}}\right) \delta_{l_{1}^{\prime} l_{1}} \delta_{l_{2}^{\prime} l_{2}}-\frac{V}{2}\left\{\left(\delta_{l_{1}^{\prime}, l_{1}-1}+\delta_{l_{1}^{\prime}, l_{1}+1}\right) \delta_{l_{2}^{\prime} l_{2}}\right.\right.} \\
\left.\left.+e^{i 2 \pi \alpha l_{1}}\left(\delta_{l_{2}^{\prime}, l_{2}-1}+\delta_{l_{2}^{\prime}, l_{2}+1}\right) \delta_{l_{1}^{\prime} l_{1}}\right\}\right] \delta_{k^{\prime} k} \\
=F_{1} l_{1} \delta_{l_{1}^{\prime} l_{1}} \delta_{l_{2}^{\prime} l_{2}}\left(\delta_{k^{\prime}, k-1}+\delta_{k^{\prime}, k+1}\right)
\end{gathered}
$$

where $F_{1}=\frac{1}{2} e a F$. To diagonalize these matrices for systems with comparable size to devices, requires a huge computational infrastructure. For one-dimensional arrays the direct diagonalization is feasible, but for a two-dimensional array, the computational cost increases very fast. In a previous paper[8] we have developed a renormalization process 
applied to a truncated Floquet matrix in a tridiagonal form with dimension $L_{1} \times L_{2} \times(2 K+1)$. The diagonal elements are formed by $\mathrm{E}^{(k)}=(\mathcal{E}-k \hbar \omega) \mathrm{I}+H_{0}$ which are a $L_{1} \times L_{2}$ block matrix representing a photon replica with the elements given by the left-hand of Eq.(3). The interaction of the ac field with the system are described by the off-diagonal matrices $\mathcal{F}^{(k)}=F_{1} l_{1} \delta_{l_{1}^{\prime} l_{1}} \delta_{l_{2}^{\prime} l_{2}}$. And we defined the Floquet Matrix as $\mathrm{M}_{\mathrm{F}}=\sum_{k} \mathrm{E}^{(k)}-\mathcal{F}^{(k)}=0$, this must be diagonalized doing $\operatorname{det}\left(\mathrm{M}_{\mathrm{F}}\right)=0$. We reduce the problem to $L_{1} \times L_{2}$ dimension by means of renormalization procedure that defines the Green function $G$ as $M_{F} G=I$, and since the Floquet states form a complete basis, the Green functions are given by

$$
\sum_{k, k^{\prime}}\left\langle l_{1}, l_{2}, k^{\prime}\left|\mathrm{M}_{\mathrm{F}}\right| l_{1}, l_{2}, k\right\rangle G_{k^{\prime}, k}=\delta_{k^{\prime}, k}
$$

We redefine the quasienergy from $\mathcal{E} \rightarrow \mathcal{E}+i \beta$, with $\beta \rightarrow 0$, and after successive "renormalization" of the Floquet matrix $M_{F}[8]$, we obtain the Green's function. From this last result, a quasi-density-of-Floquet-states, $\rho(\mathcal{E} \rightarrow \mathcal{E}+i \beta)$ can be defined as

$$
\rho(\mathcal{E}+i \beta)=-\frac{1}{\pi} \operatorname{Im}\left[\operatorname{Tr} G_{M M}\right] .
$$

The lattice models may be useful for emulating the lower part of the electronic structure of a device, that is well described by the effective-mass approximation. We choose the tight binding hopping parameter in order to emulate the lower conduction band of GaAs material with $m^{*}=0.067 m_{e}$, resulting $V=-\hbar^{2} / 2 m^{*} a^{2}=-0.142 \mathrm{eV}$, considering $a=20 \AA$. An antidot unit cell size of $a^{\prime} \times a^{\prime}=5 a \times 5 a$, and the total antidots array size of $L_{1} \times L_{2}=5 a^{\prime} \times 5 a^{\prime}=25 a \times 25 a=500 \AA \times 500 \AA$ are considered in our calculations. The size of our device is an order of magnitude smaller respect to devices experimentally obtained but our results are easily projected to actual sizes, if we consider the extreme quantum limit in the analysis[9, 10].

First, we calculate the quasi-density-of-states (DOS) as a function of $\alpha=\Phi / \Phi_{e}=p / q$ for a $14 \times 14$ square lattice with the same atomic energy $\varepsilon=4|V|=0.568 \mathrm{eV}$ for all sites and verify the first quarter of the Hofstadter butterfly for the host lattice. We apply an ac field with energy photon of $\hbar \omega=$ $50 \mathrm{meV}$ and a low field intensity of $e a F=10 \mathrm{meV}$ and $k=$ 10 photons, which produces a ratio of $e a F / \hbar \omega=0.20$. The resulting dressed spectra at high magnetic field in the region considered is shown in Fig.1. Some regions are darker than other and represent the evolution of logarithm of DOS from low values for clear regions to high values for dark regions. We can observe clearly the Hofstadter butterfly as the darkest regions, while the replicas due to one, two and three photons are decreasingly less dark, as expected.

When the ac field intensity increases to high values, the ratio of $e a F / \hbar \omega$ increases from value show in Fig. 1 to values near to zeros of the zero order Bessel function, $J_{0}(x)$ $(x=2.4048=e a F / \hbar \omega)$, and we observe that miniband widths are reduced to zero. This effect of the intense ac field is known as "dynamic localization" $[11,12]$ and has been investigated numerically in superlattices and quantum dots. Here we report that the Hosftadter spectrum also localizes dynamically. One example of this process is show in Fig. 2, when $e a F / \hbar \omega=1.0$. Here, we clearly observe modulations and collapses of the minibands at $\Phi / \Phi_{e}=0.27,0.31$ and 0.36 . The positions of the zeroes of $J_{0}$ are defined by the magnetic commensurability.

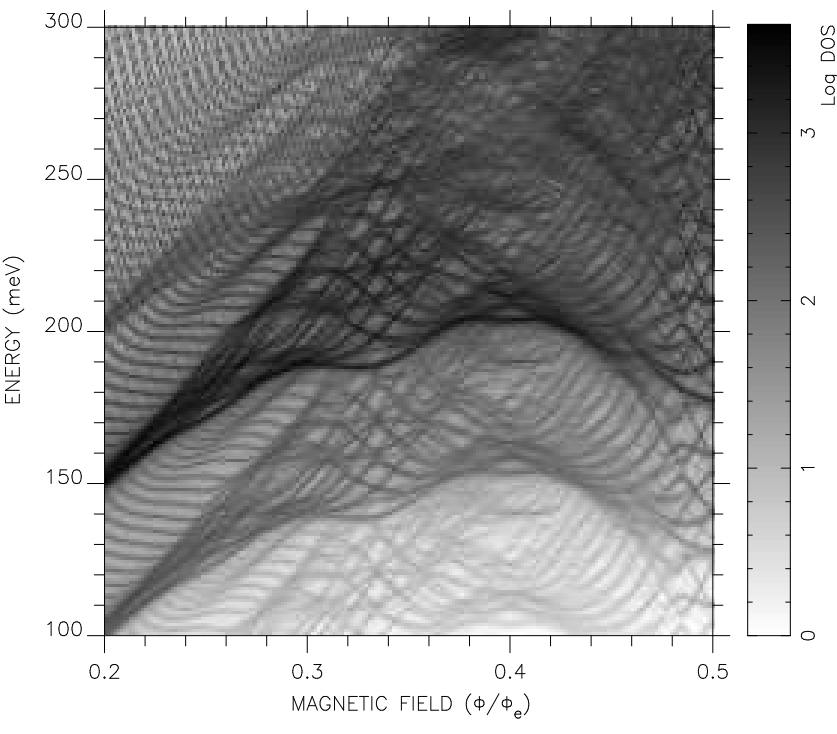

FIG. 1. Dressed Hofstadter spectra for a $14 \times 14$ square host lattice at low intensity ac field, $e a F / \hbar \omega=0.2$.

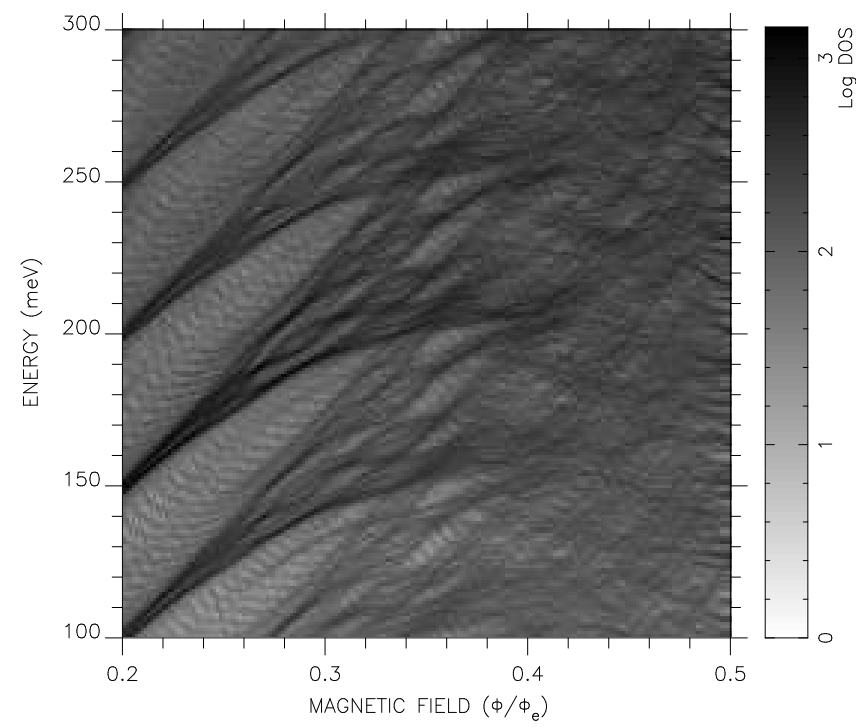

FIG. 2. Dressed Hofstadter spectra for a $14 \times 14$ square lattice at high intensity ac field, $e a F / \hbar \omega=1.0$. 


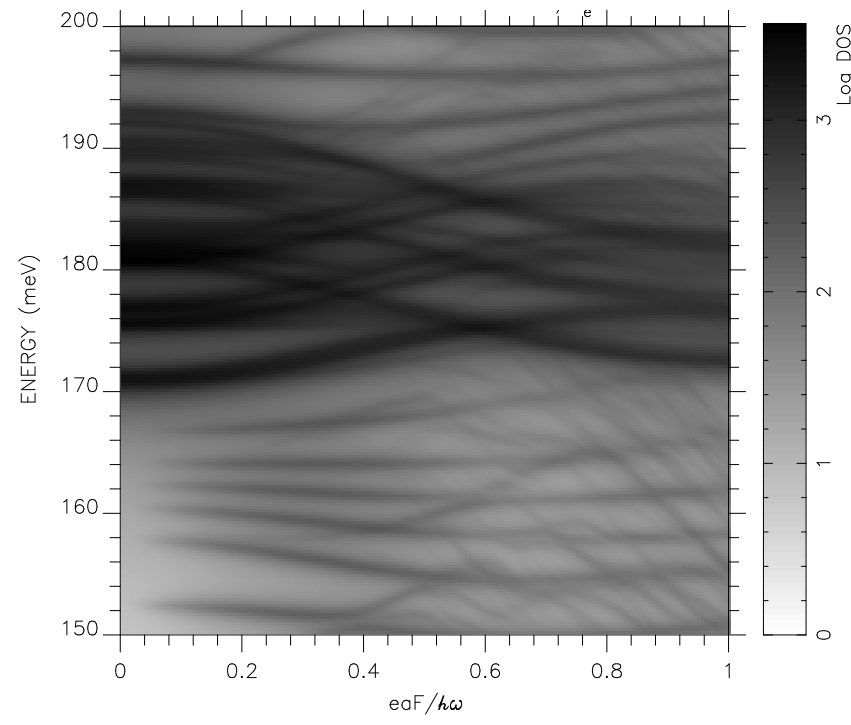

FIG. 3. Dynamical localization of dressed Hofstadter spectra for a $14 \times 14$ square lattice at $p / q=1 / 4$.

Prior to the total collapse of the Hofstadter spectra, others collapses occur in the inner structure of the Landau levels. When we observe the evolution of the spectra at $p / q=1 / 4$, while the intensity ac field is increased, the DL of the spectra for a ratio of $e a F / \hbar \omega=0.6\left(e \ell F / \hbar \omega=2.4, \ell=a \frac{q}{p}\right)$ can be seen as shown in Fig. 3.

To visualize the DOS profile when the DL occurs, we take a segment of the Fig. 3, for two special values of $e a F / \hbar \omega=0.4$ and 0.6 -without and with DL, respectively-, which are shown in Fig. 4. If we associate the value $e a F / \hbar \omega=0.6$ to $\mathrm{DL}$ of $J_{0}$, we find that this localization is associated to $2.4 / 0.6=4$, the band number $q=4$. Another localization, not show here, found at $p / q=1 / 3$ and $e a F / \hbar \omega=0.8$ means that $2.4 / 0.8=$ 3 , the band number $q=3$.

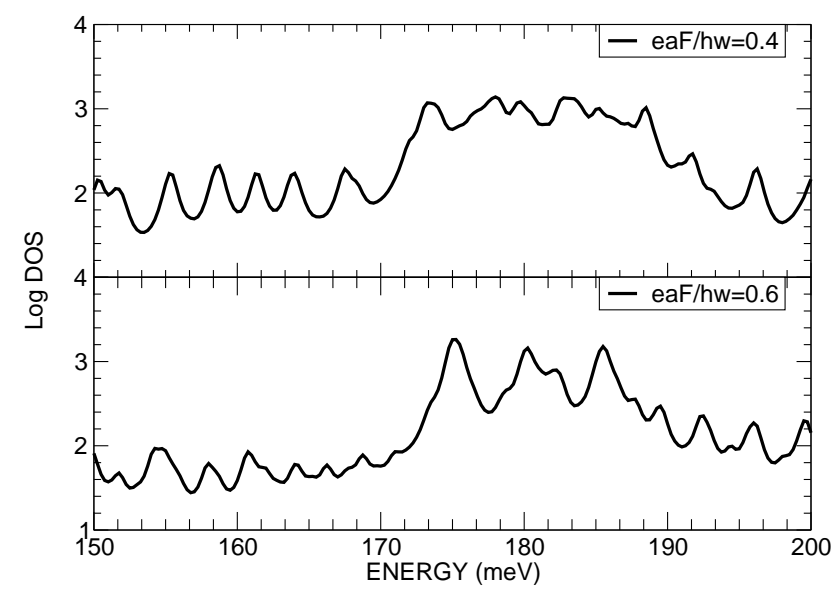

FIG. 4. DOS profile for the dynamical localization of dressed Hofstadter spectra at $p / q=1 / 4$ and $e a F / \hbar \omega=0.4$ and 0.6 , respectively. The localization occurs in the second case.

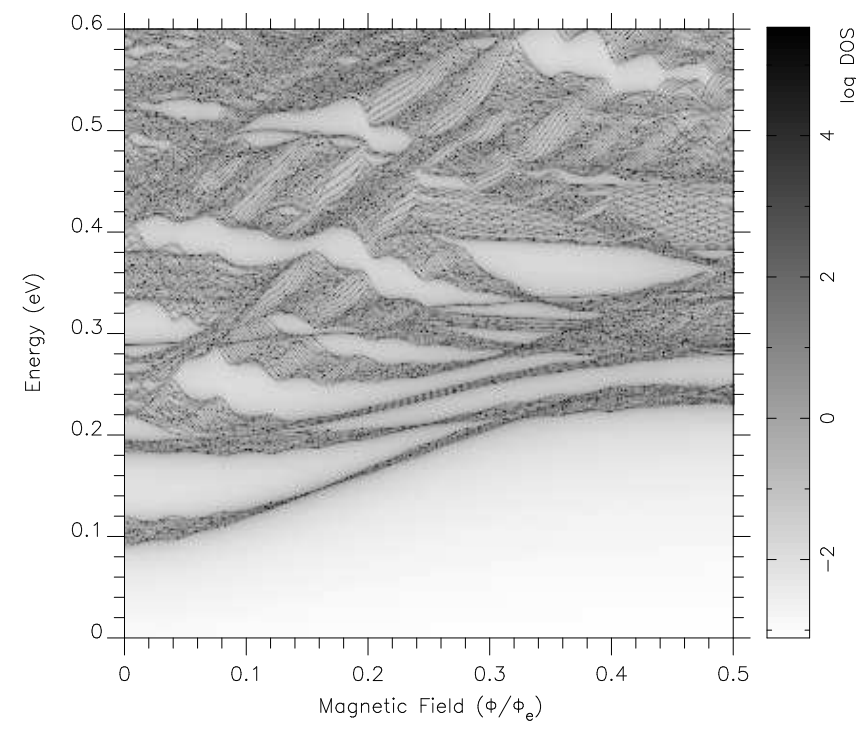

FIG. 5 . DOS of a $5 \times 5$ antidot array spectra.

Second, we calculate the quasi-DOS as function of $p / q$ for a $5 \times 5$ antidot array, each antidot with $5 \times 5$ atomic sites with energy $\varepsilon=4|V|$ and $8|V|$ describing a periodic potential modulation. This periodic modulation breaks the degenerancies of states and coupling bulk and surfaces states of dots formed by periodic potential modulation as was described in detail in Ref.[6]. The DOS of antidot array is shown in Fig. 5.

Apparently there are no traces of Hofstadter butterfly, but this spectrum is the initial stage of a $5 \times 5$ antidot array Hofstadter spectrum and, observing the inner structure of each miniband, we can see an autosimilar Hofstadter butterfly for very low magnetic fields as is shown in Fig.6. These states belong to bulk states of a quantum dot array.

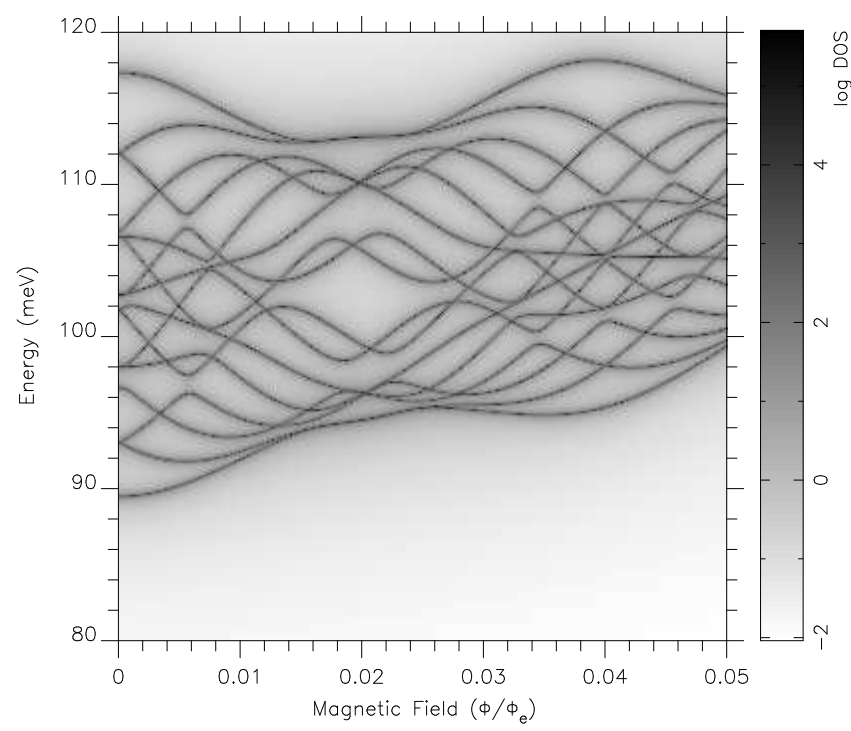

FIG. 6. DOS of first bulk-like band of a $4 \times 4$ quantum dot array. 


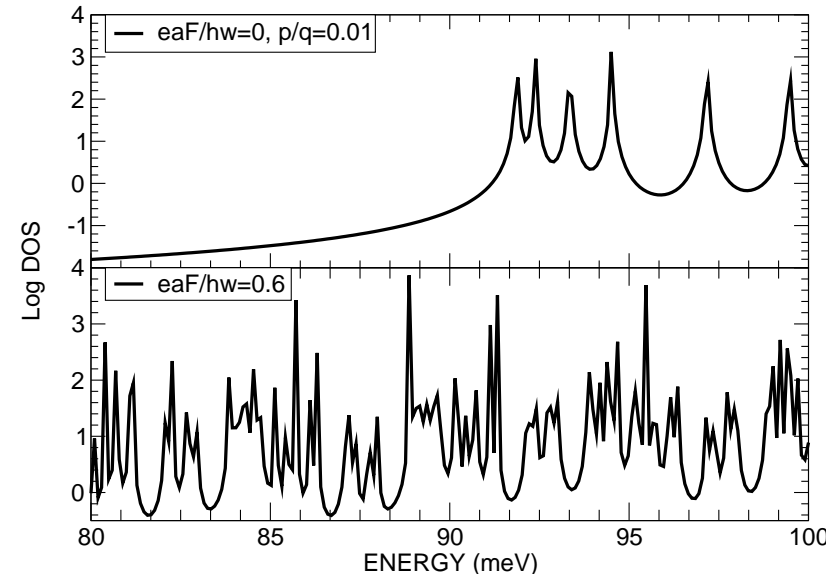

FIG. 7. Dynamical localization of autosimilar Hosftadter spectra of $4 \times 4$ quantum dot array.
The complete Hofstadter spectra are periodic with magnetic field intensity. One period finish at $p / q=0.04$, this value is 25 times lower than $p / q=1$ and the DL observed for $e a F / \hbar \omega=$ 0.6 and 0.8 must be observed in the autosimilar spectra of bulk spectra of quantum dot array formed by the antidot lattice. In Fig. 7, we show the dynamic localization of these minibands at $p / q=1 /(4 \times 25)$. Now the ac field intensity should be very low, $e a F=3 \mathrm{meV}$, as well as the energy photon, $\hbar \omega=5 \mathrm{meV}$, to reach the DL.

In conclusion, these preliminary results suggest the existence of dynamical localization in dressed Hofstadter spectra induced by an intense ac field and his effects on the formation of ZR states will be addressed in a future article.

P. H. R. acknowledges to FUNCAMP the partial financial support for the research stage in Unicamp.
[1] K. v. Klitzing, G. Dorda, and M. Pepper, Phys. Rev. Lett. 45, 494 (1980).

[2] R. G. Mani, J. H. Smet, K. von Klitzing, V. Narayanamurti, W. B. Johnson, and V. Umansky, Nature 420, 646 (2002).

[3] M. A. Zudov, R. R. Du, L. N. Pfeiffer, and K. W. West, Phys. Rev. Lett. 90, 046807 (2003).

[4] J. Dietel, L. I. Glazman, F. W. J. Hekking, and F. v. Oppen, Phys. Rev. B 71, 045329 (2005).

[5] P. H. Rivera and P. A. Schulz, Phys. Rev. B 70, 075314 (2004).

[6] P. H. Rivera, M. A. Andrade Neto, P. A. Schulz, and Nelson Studart, Phys. Rev. B 64, 35313 (2001).
[7] J. H. Shirley, Phys. Rev. 138, B979 (1965).

[8] P. A. Schulz, P. H. Rivera, and Nelson Studart, Phys. Rev. B 66, 195319 (2002).

[9] C. Albrecht, J. H. Smet, K. von Klitzing, D. Weiss, V. Umansky, and H. Schweizer, Phys. Rev. Lett. 86, 147 (2001).

[10] T. Schlosser, K. Ensslin, J. P. Kotthaus, and M. Holland, Europhys. Lett. 33, 683 (1996).

[11] D. H. Dunlap and V. M. Kenkre, Phys. Rev. B 34, 3625 (1986).

[12] M. Holthaus, Phys. Rev. Lett. 69, 351 (1992). 